\title{
Hidradenite supurativa: relato de caso
}

Ana da Costa Cardoso, Ana Cláudia Carneiro

\section{RESUMO}

Introdução: A hidradenite supurativa é uma doença inflamatória cutânea crónica e recorrente. O diagnóstico é clínico e na maior parte das vezes tardio, com um período médio até ao diagnóstico de sete anos. Este caso constitui um desafio diagnóstico, pois difere da epidemiologia mais frequente para esta doença.

Descrição do caso: Doente do sexo masculino, de 44 anos de idade, com antecedentes de excesso de peso e fumador de 28 unidades maço/ano. Em 2008 refere aparecimento de nódulos retro-auriculares e cervicais e, posteriormente, nódulos axilares e inguinais. Durante 10 anos apresenta múltiplos episódios de infeção dos nódulos e aparecimento de novas lesões, com supuração e fistulização. É submetido a múltiplos tratamentos antibióticos e a vários procedimentos excisionais. Em 2018 é levantada a hipótese diagnóstica de hidradenite supurativa, tendo iniciado antibioterapia dirigida. O diagnóstico é confirmado por dermatologia, tendo sido proposto para tratamento biológico, por falha de resposta à terapêutica antibiótica.

Comentário: O diagnóstico atempado da hidradenite supurativa é fundamental e os médicos de família possuem uma posição privilegiada para a sua abordagem inicial e referenciação atempada.

Palavras-chave: Hidradenite supurativa; Acne inversa; Glândulas apócrinas; Dermatologia.

\section{INTRODUÇÃO}

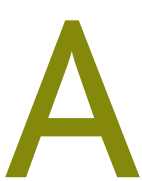
hidradenite supurativa é uma doença inflamatória cutânea crónica e recorrente, caracterizada pela existência de nódulos, abscessos e fístulas dolorosas. As lesões afetam áreas anatómicas com glândulas sudoríparas apócrinas, como axilas, virilhas, nádegas e região inframamária. ${ }^{1-6}$

A hidradenite supurativa afeta mais frequentemente mulheres (rácio de género feminino/masculino de 3:1), podendo surgir em qualquer altura entre a puberdade e a idade adulta. ${ }^{1-2}$ A prevalência Europeia estimada ronda os $1-4 \%$, sendo que a presença de antecedentes familiares, o tabagismo e o excesso de peso/obesidade são fatores de risco para o seu desenvolvimento. ${ }^{1-3,7}$

O diagnóstico é clínico, baseado em três critérios (lesões típicas: nódulos e abcessos mais ou menos profundos; localização típica e simetria bilateral - a doença tem de estar presente em uma ou mais zonas: axilas, pregas inframamárias, virilhas, ou nádegas; evolução típica: caráter crónico e/ou recorrente das lesões), pelo que muitas vezes é tardio, podendo haver um período de até sete anos para se chegar ao diagnóstico correto. ${ }^{1,3,8-9}$

*USF Lauroé, ACeS Central, ARS Algarve.
Um estudo efetuado pelo Centro de Investigação e Serviços de Saúde (CINTESIS) concluiu que a hidradenite supurativa está subdiagnosticada e subtratada em Portugal. ${ }^{10}$

O tratamento é baseado na severidade da doença. Esta pode ser classificada de acordo com o sistema de Hurley (dividida em três estádios, considerando o número de abcessos e tipo de fistulização) ou pelo Sistema Internacional de Pontuação e Gravidade da Hidradenite Supurativa, tendo em conta o número de nódulos, o número de abcessos e o número de trajetos fistulosos drenantes. ${ }^{1,4-5}$

O tratamento médico da hidradenite supurativa ativa inclui terapêutica antibiótica tópica e sistémica e terapêutica biológica. Os procedimentos cirúrgicos estão aconselhados na fase não inflamatória da doença. ${ }^{1,4-5}$

Com este trabalho, os autores pretendem descrever um caso clínico relativo a uma patologia que, não sendo rara, tem um diagnóstico tardio e difícil. Pretende-se descrever a etiologia, manifestações clínicas, diagnóstico e abordagem terapêutica breve da hidradenite supurativa. É ainda objetivo alertar os médicos de família para o diagnóstico de hidradenite supurativa e a sua referenciação atempada. 


\section{DESCRIÇÃO DO CASO Identificação e história pessoal}

Descreve-se o caso de um homem de 44 anos de idade, raça caucasiana, com doze anos de escolaridade. Atualmente trabalha como jardineiro, anteriormente desempenhava funções como eletricista.

Como antecedentes pessoais relevantes, foi submetido a amigdalectomia na infância e fraturou o fémur no seguimento de um acidente de viação aos 17 anos, com necessidade de tratamento cirúrgico e transfusão sanguínea. Sem utilização de medicação crónica. Nega a existência de alergias medicamentosas ou alimentares. Fumador de 28 unidades maço/ /ano, sem hábitos alcoólicos ou toxicológicos conhecidos e com um estilo de vida sedentário. Apresenta excesso de peso, com um índice de massa corporal de $26,6 \mathrm{Kg} / \mathrm{m}^{2}$. Tem o Plano Nacional de Vacinação atualizado.

\section{Caracterização familiar}

O utente integra uma família monoparental e de classe socioeconómica III (média), de acordo com a classificação de Graffar. É divorciado há três anos e reside com os três filhos do género masculino. Apresenta-se o genograma desta família e o respetivo agregado familiar (Figura 1), segundo informação fornecida pelo próprio.

\section{História da doença atual}

\section{Março de 2008}

O utente referiu aparecimento de dois nódulos cer-

Figura 1. Genograma Familiar.

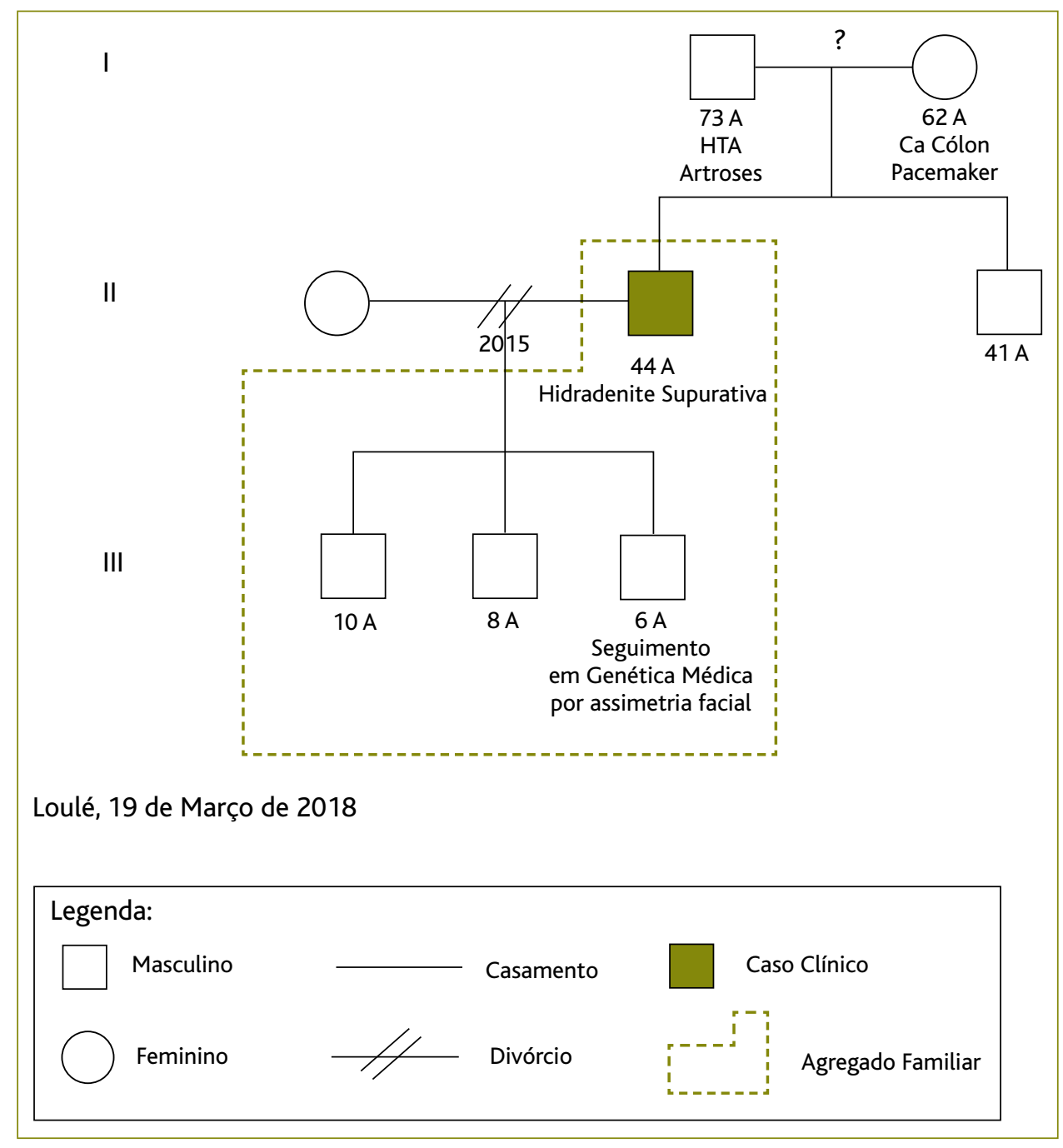

vicais e três retro-auriculares à direita, com cerca de $1 \mathrm{~cm}$ de maior diâmetro, bordos regulares, consistência mole, sem aderência a planos profundos, com rubor, calor, edema e dor associados, tendo recorrido a clínica privada, onde foram removidos cirurgicamente. Alguns meses depois apresentou lesões nodulares nas axilas e virilhas, tendo sido medicado com antibióticos sistémicos e tópicos, que não sabe especificar.

\section{Abril de 2011}

Até esta data, o utente referiu seguimento irregular em consulta privada com realização de tratamentos antibióticos, que não sabe especificar. Nesta altura 
recorreu a consulta no seu médico de família e, por apresentar lesões nodulares axilares bilaterais residuais, indolores e sem outros sinais inflamatórios associados, foi referenciado a consulta de cirurgia do hospital de referência. Devido ao atraso na resposta, decidiu recorrer a uma instituição privada, onde fez excisão de nódulos axilares bilateralmente.

\section{Fevereiro de 2013}

Recorreu a consulta no seu médico de família por apresentar nódulo na região axilar esquerda com supuração contínua, associado a queixas álgicas. Foi prescrita antibioterapia com cefuroxima 500mg de $12 / 12 \mathrm{~h} \mathrm{du}-$ rante oito dias e efetuado novo pedido de referenciação para consulta de cirurgia do hospital de referência.

\section{Abril de 2014}

Foi avaliado em consulta de cirurgia. Nesta altura, para além de manter o nódulo axilar, agora sem sinais inflamatórios, apresentava outro nódulo na região nadegueira direita, doloroso, com rubor, calor e edema local e da região circundante à lesão, mas sem menção de tamanho no registo clínico, tendo sido diagnosticado com abcesso e celulite da nádega direita. Foi referenciado para excisão da lesão em pequena cirurgia ambulatória e foi dada alta da consulta externa de cirurgia, sem menção de instituição de antibioterapia.

\section{Junho de 2014}

Em consulta com o seu médico de família e, por apresentar novo nódulo axilar à direita, com supuração, foi instituída medicação antibiótica com amoxicilina e ácido clavulâmico 875+125mg de 12/12h durante oito dias e efetuada nova referenciação para a consulta de cirurgia.

\section{Fevereiro de 2015}

Avaliado em consulta de cirurgia do hospital de referência, por apresentar quisto sebáceo axilar com múltiplos episódios de fistulização, já com retração cutânea e vários orifícios de drenagem, foi referenciado para cirurgia plástica do mesmo hospital, para a qual nunca chegou a ser convocado.

\section{Fevereiro de 2017}

Recorre ao seu médico de família por apresentar fístula na região internadegueira, com drenagem de con- teúdo purulento, associada a rubor, calor e edema locais. Foi medicado com amoxicilina e ácido clavulâmico $875+125 \mathrm{mg}$ de $12 / 12 \mathrm{~h}$ durante oito dias e foi efetuada nova referenciação para consulta de cirurgia do hospital de referência.

\section{Abril de 2017}

Avaliado em consulta de cirurgia por fistulização do sulco nadegueiro, com drenagem escassa. Foi agendada nova consulta para excisão da lesão em ambulatório.

\section{Janeiro de 2018}

Excisão de nódulo em hospital privado através do cheque cirurgia, emitido pelo seu hospital de referência.

\section{Fevereiro de 2018}

Recorreu ao seu centro de saúde para realização de penso a lesões axilares e foi solicitada avaliação médica pela equipa de enfermagem. Apresentava toda a região axilar bilateralmente com lesões abcedadas dolorosas, com drenagem purulenta e zonas de fistulização, com rubor, calor e edema, levantando-se a suspeita diagnóstica de hidradenite supurativa [foi inserido diagnóstico no S. Clínico: Doença das Glândulas Sudoríparas (S92)]. Iniciou antibioterapia com clindamicina $300 \mathrm{mg}$ de $12 / 12 \mathrm{~h}$ associada a rifampicina $300 \mathrm{mg}$ de $12 / 12 \mathrm{~h}$, que deveria manter durante 12 semanas.

Tendo em conta a equipa restrita do serviço de dermatologia do hospital de referência, com capacidade de resposta apenas para casos de suspeita de neoplasia, optou-se pelo contacto telefónico para tentar a referenciação do caso (já que o doente não pretende referenciação para outro hospital, por dificuldade em deslocações fora da região).

\section{Março de 2018}

Após autorização do serviço de dermatologia procedeu-se à referenciação deste doente. Numa das suas visitas ao centro de saúde para realização de penso e, após o seu consentimento informado, foi efetuado o registo fotográfico das lesões (Figura 2) e feito o pedido de consulta via teledermatologia através do AlertP1.

\section{Junho de 2018}

Primeira avaliação em consulta de dermatologia do hospital de referência, tendo sido confirmado o 

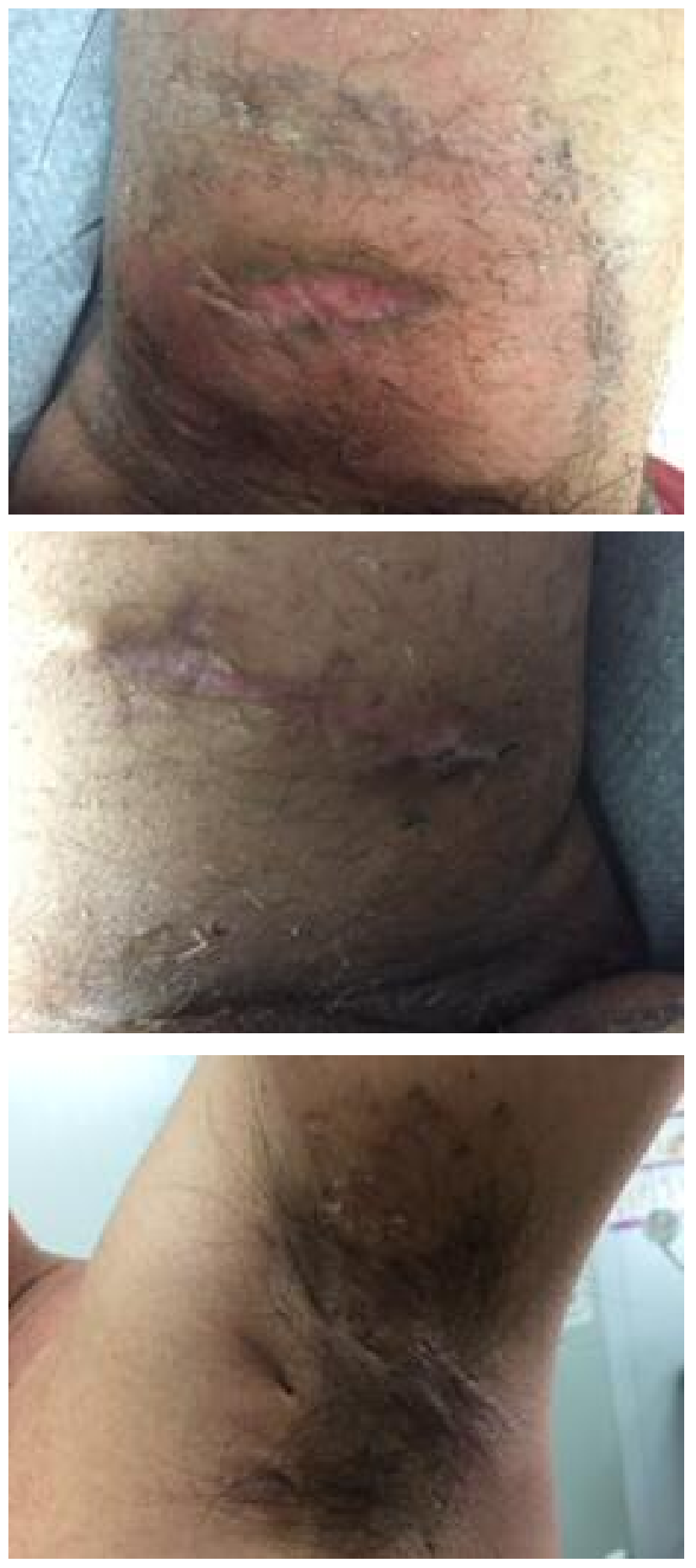

Figura 2. Registo fotográfico das lesões.

diagnóstico de hidradenite supurativa Hurley III.

Foi estabelecido um plano terapêutico com o doente, com necessidade de cessação tabágica e início de doxiciclina 100mg, uma toma por dia durante três meses. Foi solicitada avaliação analítica com hemograma, transaminases, perfil lipídico, função renal, serologias para hepatite B e C e vírus da imunodeficiência humana e radiografia torácica para início de terapêutica biológica, tendo ficado agendada reavaliação em três meses.

\section{Setembro de 2018}

Avaliado em consulta de seguimento de dermatologia. Sem alteração dos resultados dos exames complementares de diagnóstico solicitados na consulta anterior. Assinou termo de responsabilidade e consentimento informado para o início de terapêutica biológica com adalimumab e foi proposta a técnica de Deroofing para as lesões axilares. A Figura 3 resume os principais acontecimentos relatados.

\section{COMENTÁRIO}

A propósito deste caso abordam-se alguns pontos de reflexão.

A hidradenite supurativa constitui um enorme desafio diagnóstico, dada a natureza inespecífica dos seus sintomas iniciais.

Esta doença tem impacto consideravelmente negativo na qualidade de vida dos indivíduos, devido à dor e odor associados às lesões, o que leva ao estigma social e ao isolamento. A doença tem também um impacto emocional negativo, associado a depressão e alterações na vida sexual. As taxas de absentismo laboral nestes doentes são mais elevadas comparativamente à população em geral. ${ }^{1,3,5,8-9}$

Este caso difere da epidemiologia mais frequente da hidradenite supurativa, já que se trata de um homem, sendo esta patologia mais comum no género feminino; com aparecimento inicial de nódulos cervicais e retro-auriculares (não sendo estes os locais mais frequentes de manifestação da doença) e só posteriormente com afeção das regiões axilares e inguinais, o que inicialmente pode ter limitado a associação à hidradenite supurativa. Contudo, este homem possuía os fatores de risco associados a esta doença, era fumador e apresentava excesso de peso.

Não se trata de um caso inédito de atraso de diagnóstico (apesar de neste caso existir um atraso de 10 anos, o que é superior à média), ${ }^{1,3,9}$ mas antes da 


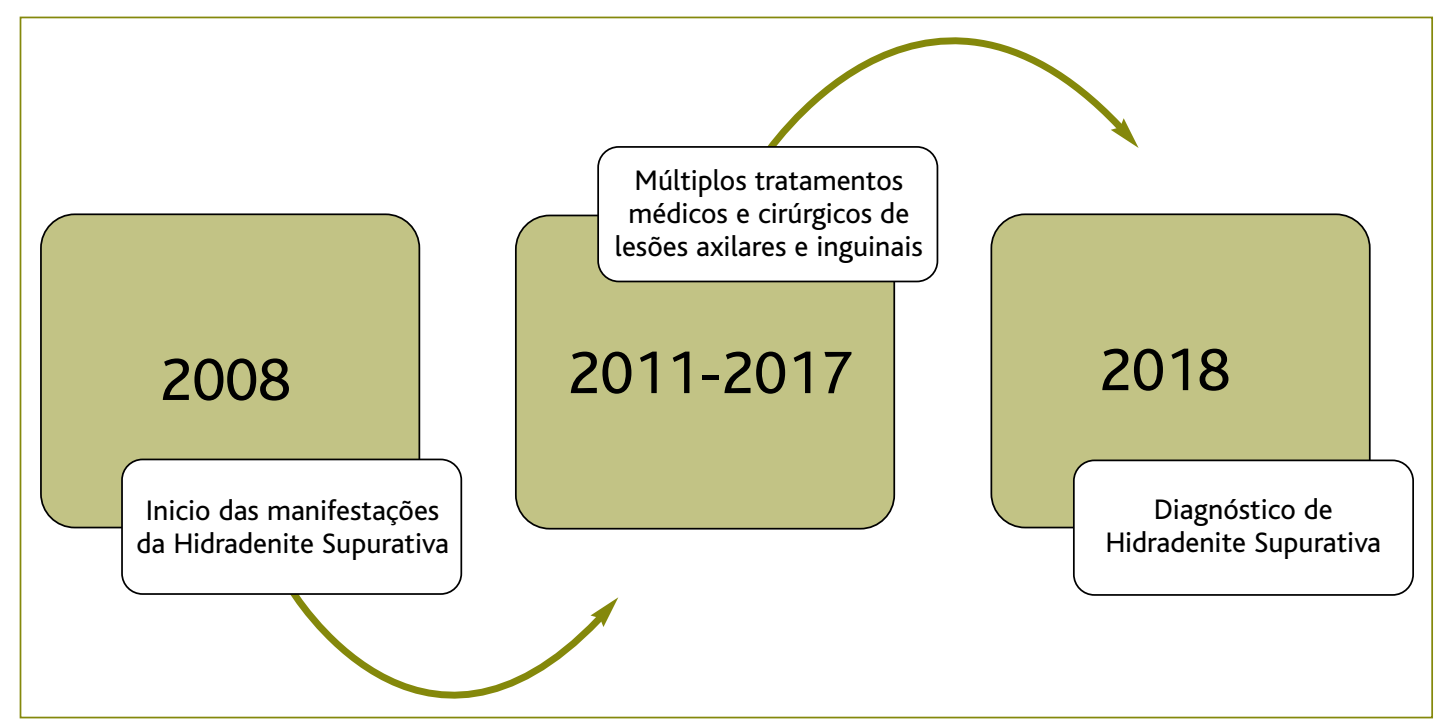

Figura 3. Resumo dos principais acontecimentos.

divulgação de um exemplo rico, que demonstra o percurso típico de um doente com hidradenite supurativa e, por isso, um alerta aos profissionais de saúde para estarem despertos para os sinais e sintomas inerentes a esta doença.

Verificou-se também uma limitação em termos da acessibilidade aos cuidados de saúde secundários, com tempos de espera elevados, o que contribuiu ainda mais para o atraso de diagnóstico.

A referenciação para dermatologia nas situações com lesões nodulares, com processos intermitentes de infeção que se mantêm ao longo do tempo, é fundamental e não deve ser protelada no tempo.

Esta patologia deve ter uma abordagem multidisciplinar, com vigilância dermatológica e cirúrgica, pois ao longo da sua evolução e estadio necessita de diferentes tipos de intervenção. É por esta razão que o médico de família tem um papel de relevo dada a sua posição privilegiada e visão abrangente dos sintomas e da sua evolução. A sua perceção da cronicidade dos sintomas e da dificuldade na gestão da doença são uma mais valia na possibilidade da referenciação e do diagnóstico atempados.

Após o diagnóstico, o médico de família será também o melhor intermediário do doente nas diferentes fases da sua doença, entre as especialidades de dermatologia e cirurgia, tendo também um papel de fulcral im- portância na redução dos fatores de risco associados, como a cessação tabágica, a promoção de estilos de vida saudáveis (tendo em vista a manutenção de um peso saudável) e na gestão do impacto psicossocial que esta doença acarreta.

\section{REFERÊNCIAS BIBLIOGRÁFICAS}

1. Jemec GB. Hidradenitis suppurativa. N Engl J Med. 2012;366(2):15864.

2. Collier F, Smith RC, Morton CA. Diagnosis and management of hidradenitis suppurativa. BMJ. 2013;346:f2121.

3. Dufour DN, Emtestam L, Jemec GB. Hidradenitis suppurativa: a common and burdensome, yet under-recognised, inflammatory skin disease. Postgrad Med J. 2014;90(1062):216-21.

4. Gulliver W, Zouboulis CC, Prens E, Jemec GB, Tzellos T. Evidence-based approach to the treatment of hidradenitis suppurativa/acne inversa, based on the European guidelines for hidradenitis suppurativa. Rev Endocr Metab Disord. 2016;17(3):343-51.

5. Zouboulis CC, Desai N, Emtestam L, Hunger RE, loannides D, Juhász I, et al. European S1 guideline for the treatment of hidradenitis suppurativa/acne inversa. J Eur Acad Dermatol Venereol. 2015;29(4):619-44.

6. Shahi V, Alikhan A, Vazquez BG, Weaver AL, Davis MD. Prevalence of hidradenitis suppurativa: a population-based study in Olmsted County, Minnesota. Dermatology. 2014;229(2):154-8.

7. Vazquez BG, Alikhan A, Weaver AL, Wetter DA, Davis MD. Incidence of hidradenitis suppurativa and associated factors: a population-based study of Olmsted County, Minnesota. J Invest Dermatol. 2013;133(1):97103.

8. Revuz JE, Jemec GB. Diagnosing hidradenitis suppurativa. Dermatol Clin. 2016;34(1):1-5. 
9. Napolitano M, Megna M, Timoshchuk EA, Patruno C, Balato N, Fabbrocini $G$, et al. Hidradenitis suppurativa: from pathogenesis to diagnosis and treatment. Clin Cosmet Investig Dermatol. 2017;10:105-15.

10. Santos JV, Lisboa C, Lanna C, Costa-Pereira A, Freitas A. Is the prevalence of hidradenitis suppurativa being overestimated in Europe? Or is the disease underdiagnosed? Evidence from a nationwide study across Portuguese public hospitals. Int J Dermatol. 2017;56(12): 1491-2.

\section{CONFLITO DE INTERESSES}

As autoras declaram não existir conflitos de interesse relacionados com a realização/publicação deste relato de caso. Os custos associados à realiza- ção deste artigo foram suportados pelas próprias. Foi obtido o consentimento informado, livre e esclarecido, dado por escrito.

\section{ENDEREÇO PARA CORRESPONDÊNCIA \\ Ana da Costa Cardoso \\ E-mail: analucia.costa.cardoso@gmail.com \\ https://orcid.org/0000-0002-2281-9258}

Recebido em 26-10-2018

Aceite para publicação em 07-05-2019

\section{ABSTRACT}

\section{SUPPURATIVE HIDRADENITIS: CASE REPORT}

Introduction: Hidradenitis suppurativa is a recurrent chronic inflammatory skin disease. The diagnosis is clinical and most often late - the average period of diagnosis is seven years. This case presents a diagnostic challenge since it differs from the most frequent epidemiology for this disease

Case description: Male patient, 44 years of age, with a history of overweight and smoker of 28 pack/year. In 2008 , the patient refers to the appearance of retro auricular and cervical nodules and later, axillary and inguinal nodules. For 10 years, the patient presents multiple episodes of infection of the nodules and appearance of new lesions, with suppuration and fistulization. The patient was subjected to multiple antibiotic treatments and various excisional procedures. In 2018 the diagnostic hypothesis of hidradenitis suppurativa was raised and initiated targeted antibiotic therapy. The diagnosis is confirmed by dermatology and the patient has been proposed for biological treatment, due to the failure of the antibiotic therapy.

Commentary: The timely diagnosis of hidradenitis suppurativa is essential and family physicians have a privileged position for the initial approach and timely referral.

Keywords: Hidradenitis supurativa; Acne inversa; Apocrine glands; Dermatology. 